\title{
The hydrophilic/hydrophobic nature of a Cl-terminated Si surface
}

\author{
P. Chatterjee, ${ }^{a}$ and S. Hazra, ${ }^{* a}$
}

The hydrophilic/hydrophobic nature of a Cl-terminated Si surface, which is slightly controversial, was investigated on the molecular level by depositing CTAB-silica 2D-hexagonal mesostructured films on it as well as on OH- and H-terminated Si surfaces and determining their structures by combining X-ray reflectivity and grazing incidence small angle X-ray scattering measurements. The contact angle measurements, which provide macroscopic level information, confirm that the Cl-Si surface is hydrophilic in nature but less hydrophilic than the $\mathrm{OH}-\mathrm{Si}$ surface. The initial attachment of a film (through a silica layer) and the (near circular) shape of the micelles, which provide microscopic level information, clearly indicate that the Cl-Si surface is hydrophilic in nature and its hydrophilicity is comparable to that of the $\mathrm{OH}-\mathrm{Si}$ surface. The relatively strong electronegativity of the $\mathrm{Cl}$ is probably the reason for the hydrophilic nature of the $\mathrm{Cl}$-Si surface, while the lack of hydrogen bonding and/or the lack of complete chlorination are a hindrance.

\section{Introduction}

Silicon is perhaps the most widely investigated semiconductor material due to its various uses in device fabrication. Among different investigations, one of the thrust areas is tuning the nature of $\mathrm{Si}$ surface to control the growth and stability of different interesting nanostructures on it. ${ }^{1-9}$ Tuning the nature of the surface can be done through the termination or passivation of the Si dangling bonds with selective atoms or groups, which essentially modifies the surface free energy, the polar-nonpolar (hydrophilic-hydrophobic) or electrostatic nature, the surface defect states and the reactivity of the surface. $^{5-13}$

Halogen-terminated Si surface were found to be more reactive compared to H-terminated Si surface in functionalization reactions ${ }^{14,15}$ and can facilitate the growth of materials with a high dielectric constant, the attachment of self-assembled monolayers, or the production of simple organic layers for application in biotechnology. ${ }^{15,16}$ In particular, alkyl Grignards react readily with a chlorinated Si surface to create an alkyl-terminated Si surface. The efficient assembly of organic molecules on Si using the S-N linkage was achieved by reacting amines with a Cl-terminated $\mathrm{Si}$ surface. ${ }^{17}$ Such $\mathrm{Cl}-\mathrm{Si}$ surfaces, which have good $\mathrm{Si}-\mathrm{Cl}$ bond stability, were recently prepared from the $\mathrm{H}-\mathrm{Si}$ surfaces using different methods in ambient conditions, which is much more applicable to industrial purposes. Among them, the wet-chemical process is one of the best and easiest methods to maintain a contaminationfree Si substrate, and is drawing tremendous attention. ${ }^{18-20}$

Despite a large number of works on the Cl-Si surface, only a limited number of works has been carried out to determine the hydrophobic/hydrophilic nature of the surface, ${ }^{21-23}$ which again provides contradictory or incomplete information about the nature of the surface, at least on the microscopic level. For example, the macroscopic contact angle measurement for the $\mathrm{Cl}-\mathrm{Si}$ surface was found to be small compared to that of the

${ }^{a}$ Saha Institute of Nuclear Physics, 1/AF Bidhannagar, Kolkata 700064, India.E-mail: satyajit.hazra@saha.ac.in hydrophobic H-Si surface. ${ }^{21}$ This along with ultrafast electron crystallography experiments on the adsorbed water molecules suggests that the $\mathrm{Cl}-\mathrm{Si}$ surface is hydrophilic in nature. ${ }^{21} \mathrm{On}$ the other hand, a theoretical calculation on the adsorption of water on the Cl-Si surface based on a full first-principles approach suggests that the surface is hydrophobic in nature. ${ }^{22,23}$ It has thus became extremely important and essential to use other experimental techniques or schemes to determine the hydrophobic/hydrophilic nature of the $\mathrm{Cl}-\mathrm{Si}$ surface.

There are different ways to understand the hydrophilic/hydrophobic nature of a surface. A recent review article proposed different measures and classifications of the hydrophilic/hydrophobic nature of solid surfaces based on different parameters such as the contact angle, the water adhesion tension, the work of spreading or the energy of hydration. ${ }^{24}$ All these parameters are essentially related with the surface free energy and the interfacial energy. Contact angle measurements provide information on the wetting, while direct adhesion measurements using a centrifugal adhesion balance provide information on the affinity. ${ }^{25-27}$ The information on the adhesion can also be available from the frictional force microscopy.

It is known that the structure of the monolayer or multilayer film of metal-organic or organic-inorganic (having both hydrophobic and hydrophilic parts) on the pretreated Si surface strongly depends on the nature of the $\mathrm{Si}$ surface. ${ }^{7,13}$ The preferential adsorption of materials can provide information on the adhesion and the hydrophilic/hydrophobic nature of a surface. For example, the adsorption of the metalorganic film deposited by LB technique on a hydrophilic $\mathrm{OH}-$ terminated Si surface proceeds via hydrophilic metal head groups, while adsorption on a hydrophobic $\mathrm{H}$-terminated $\mathrm{Si}$ surface proceeds via hydrophobic organic tail groups. ${ }^{7}$ Similarly, for the CTAB-silica (organic-inorganic) mesostructured film, hydrophilic silica molecules are adsorbed on the $\mathrm{OH}-\mathrm{Si}$ surface to form a silica layer (gray color), while the hydrophobic tail parts of the CTAB molecules are adsorbed on the $\mathrm{H}-\mathrm{Si}$ surface to form hemicircular micelles (light color), ${ }^{13}$ as pre- 

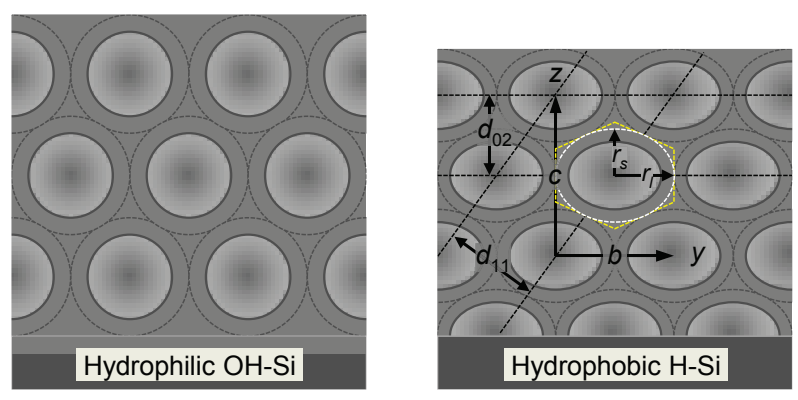

Fig. 1 Schematics of 2D-hexagonal mesostructured films on hydrodrophilic and hydrophobic Si substrates and an equivalent centered rectangular $(c 2 \mathrm{~mm})$ structure with unit cell parameters $(b$ and $c$ ), lattice spacings ( $d_{02}$ and $d_{11}$ ), the Wigner-Seitz cell (yellow dashed lines) and corresponding ellipse (white curve) with semi-major $\left(r_{l}\right)$ and semi-minor $\left(r_{s}\right)$ axis.

sented schematically in Fig. 1. Accordingly, the near circular micelles on the former became less circular (or more elliptical) on the latter (light color). It is clear that the hydropho$\mathrm{bic} /$ hydropholic nature of a solid surface, on the microscopic level, can be evident from the structural information of the deposited film.

In this paper we have used this scheme (adsorption and shape effects) to understand the hydrophobic/hydropholic nature of the Cl-Si surface. CTAB-silica 2D-hexagonal mesostructured thin films ${ }^{28-30}$ were deposited on the $\mathrm{Cl}-\mathrm{Si}$ surface and also on the $\mathrm{OH}-\mathrm{Si}$ and $\mathrm{H}-\mathrm{Si}$ surfaces. The chlorination of the $\mathrm{Si}$ surface through a wet-chemical process is verified using an X-ray photoelectron spectroscopy (XPS) technique. The hydrophilic nature of the $\mathrm{Cl}-\mathrm{Si}$ surface, on the macroscopic lever, is confirmed by conventional contact angle (CA) measurement, while the complete structures of the films were obtained by complementary X-ray reflectivity (XR) $)^{5,7,31,32}$ and grazing incidence small angle X-ray scattering (GISAXS) ${ }^{13,33-37}$ techniques, which clearly suggest that the $\mathrm{Cl}-\mathrm{Si}$ surface is hydrophilic in nature, even an the microscopic level. A possible reason behind the hydrophilic-like nature of the Cl-Si surface is discussed.

\section{Experiment}

\subsection{Preparation}

Hydrophilic and hydrophobic $\mathrm{Si}(001)$ and $\mathrm{Si}(111)$ substrates were prepared through different standard pretreatments, as reported before. ${ }^{5,7,10,11,13}$ In short, after removing the organic contaminants, ${ }^{5}$ all the substrates (of the size $15 \times 15 \mathrm{~mm}^{2}$ ) were treated with the RCA cleaning method, where the substrates were boiled at $100^{\circ} \mathrm{C}$ for about $10 \mathrm{~min}$ in a mixed solution of ammonium hydroxide $\left[\mathrm{NH}_{4} \mathrm{OH}\right.$, Merck, 30\%], hydrogen peroxide $\left[\mathrm{H}_{2} \mathrm{O}_{2}\right.$, Merck, 30\%] and Milli-Q water $\left(\mathrm{NH}_{4} \mathrm{OH}: \mathrm{H}_{2} \mathrm{O}_{2}: \mathrm{H}_{2} \mathrm{O}=1: 1: 5\right.$, by volume). The sub- strates were then rinsed thoroughly with Milli-Q water. In this process, a fresh oxide layer, which is terminated with an $\mathrm{OH}$ group, was grown on the $\mathrm{Si}$ surface after removing the native oxide layer. ${ }^{7,10,11,13}$ The $\mathrm{OH}$-terminated $\mathrm{Si}$ substrates (labelled as $\mathrm{OH}-\mathrm{Si}$ ) are hydrophilic in nature. To create $\mathrm{H}$ terminated $\mathrm{Si}$ substrates, some of the $\mathrm{OH}-\mathrm{Si}$ substrates were further etched in a solution of hydrogen fluoride [HF, Merck, $10 \%$ ] for $30 \mathrm{~s}$ at room temperature $\left(25^{\circ} \mathrm{C}\right)$. Among them, the $\mathrm{Si}(111)$ substrates were subsequently etched in an ammonium fluoride $\left[\mathrm{NH}_{4} \mathrm{~F}\right.$, Merck, 40\%] solution for $2 \mathrm{~min}$ at room temperature. ${ }^{11}$ Such H-terminated Si substrates (labelled as $\mathrm{H}-\mathrm{Si}$ ) are hydrophobic in nature.

Some of the H-Si substrates were then chlorinated through a wet-chemical method as reported in the literature. ${ }^{18}$ A stock chlorinating solution was prepared by dissolving phosphorus pentachloride $\left[\mathrm{PCl}_{5}\right.$, Merck] in chlorobenzene $\left[\mathrm{C}_{6} \mathrm{H}_{5} \mathrm{Cl}\right.$, Merck] to form a near-saturated solution (typically $0.6 \mathrm{M}$ ). The solution was heated at $\sim 60^{\circ} \mathrm{C}$ for $1 \mathrm{~h}$ for complete dissolution of the $\mathrm{PCl}_{5}$. Immediately before use, a few grains of benzoyl peroxide $(\sim 300 \mathrm{mg})$ were added to the stock solution $(\sim 80 \mathrm{ml})$. The $\mathrm{H}-\mathrm{Si}$ samples were immersed into the solution, which was heated to $90-100^{\circ} \mathrm{C}$ for $45 \mathrm{~min}$. The samples were then rinsed with anhydrous tetrahydrofuran and anhydrous methanol. This set of substrates was labelled as $\mathrm{Cl}-\mathrm{Si}$.

A silica-surfactant solution was prepared by a two step synthesis method using tetraethyl orthosilicate [TEOS, $\mathrm{Si}\left(\mathrm{OC}_{2} \mathrm{H}_{5}\right)_{4}$, Sigma Aldrich, $99.999 \%$ ], cetyltrimethylammonium bromide [CTAB, $\mathrm{C}_{16} \mathrm{H}_{33} \mathrm{~N}\left(\mathrm{CH}_{3}\right)_{3} \mathrm{Br}$, Fluka, $\geq 99 \%$ ], ethanol $\left[\mathrm{C}_{2} \mathrm{H}_{5} \mathrm{OH}\right.$, Merck, absolute] and Milli-Q water (resistivity $18.2 \mathrm{M} \Omega \mathrm{cm}$ ) and hydrochloric acid [HCl, Merck, 35\%] as reported before. ${ }^{13}$ In the first step, a silica solution was prepared with TEOS in acidic condition with molar ratio of TEOS $: \mathrm{C}_{2} \mathrm{H}_{5} \mathrm{OH}: \mathrm{H}_{2} \mathrm{O}=1: 4.5: 1$ and finally added the required amount of $\mathrm{HCl}$ to the the solution to keep the $\mathrm{pH} \approx 1$. The solution was then stirred for $1 \mathrm{~h}$ at room temperature. In parallel, a second solution was prepared by dissolving $0.348 \mathrm{~g}$ of CTAB in ethanol and water of molar ratio $3.66: 1$. Finally, the second solution was added to the first one to get the final solution with the molar ratios of TEOS : CTAB : $\mathrm{C}_{2} \mathrm{H}_{5} \mathrm{OH}$ : $\mathrm{H}_{2} \mathrm{O}=1: 0.19: 20: 5.5$, which was then stirred for another $1 \mathrm{~h}$ and aged for $2 \mathrm{~h}$. A diluted solution was then prepared by adding excess ethanol (of amount $35 \mathrm{cc}$ ) to the stock solution to prepare thin films.

Films were then prepared (at temperature: $25-30^{\circ} \mathrm{C}$ and relative humidity: 70-75\%) using a spin-coater (EC101, Headway Research) at a speed of $4000 \mathrm{rpm}$ from a diluted solution on the Cl-Si substrates and also on the $\mathrm{OH}-\mathrm{Si}$ and $\mathrm{H}-$ $\mathrm{Si}$ substrates for comparison, which were labelled as CTAB$\mathrm{SiO} / \mathrm{Cl}-\mathrm{Si}$, CTAB-SiO/OH-Si and CTAB-SiO/H-Si, respectively. Samples were then preserved at the X-ray laboratory, where temperature and relative humidity were maintained at $\sim 25^{\circ} \mathrm{C}$ and $\sim 40 \%$, respectively. 


\subsection{Characterization}

XPS measurements of the $\mathrm{Cl}-\mathrm{Si}(001)$ and $\mathrm{Cl}-\mathrm{Si}(111)$ samples were performed in a multiprobe UHV chamber (Omicron NanoTechnology) equipped with an $\mathrm{Al} \mathrm{K} \alpha$ source $(E=1486.6 \mathrm{eV})$ and a hemispherical analyzer (EA 125) to determine the chemical nature of the surfaces. The samples were transferred to the UHV chamber after preparation.

CA measurements of the $\mathrm{Cl}-\mathrm{Si}(001)$ and $\mathrm{Cl}-\mathrm{Si}(111)$ samples were carried out using CA goniometer (ramé-hart) to investigate the hydrophilic or hydrophobic nature of the Clterminated Si surfaces. For the measurement, a drop $(5 \mu \mathrm{l})$ of water (Milli-Q) was placed on the sample surface. The CA values were noted after a minute and then 5 successively times at intervals of $20 \mathrm{~s}$. Such measurements were done in more than five different regions over the sample surface. The CA value was determined by taking the average of the final measurements in the five different regions. The CA measurements of the OH-Si and H-Si samples were also carried out for comparison.

The XR measurements of the CTAB-SiO/Cl-Si, CTAB$\mathrm{SiO} / \mathrm{OH}-\mathrm{Si}$ and CTAB-SiO/H-Si samples were performed within a day and up to 30 days after preparation (dried films) on a versatile X-ray diffractometer (VXRD) setup. ${ }^{38}$ The XR measurements of the as-prepared $\mathrm{Cl}-\mathrm{Si}(001)$ and $\mathrm{Cl}-\mathrm{Si}(111)$ samples were also carried out using the VXRD setup to check the surface roughness. The VXRD setup consists of a diffractometer (D8 Discover, Bruker AXS) with a $\mathrm{Cu}$ source (sealed tube), followed by a Göbel mirror to select and enhance the $\mathrm{Cu}$ $\mathrm{K} \alpha$ radiation $(\lambda=1.54 \AA)$. The diffractometer has a two-circle goniometer $[\theta(\omega)-2 \theta]$ with a quarter-circle Eulerian cradle as the sample stage. The latter has two circular $(\chi$ and $\phi)$ and three translational (X, Y, and Z) motions. The scattered beam was detected using a NaI scintillation (point) detector. Data were recorded under specular condition, i.e. with an incident angle $\alpha$ equal to the reflected angle $\beta(\alpha=\beta=\theta)$. Under such condition there exists a nonvanishing wave vector component, $q_{z}$, which is equal to $(4 \pi / \lambda) \sin \theta$ with a resolution of $0.002 \AA^{-1}$. The XR data essentially provide an electrondensity profile (EDP), i.e., the in-plane $(x-y)$ average electron density $(\rho)$ as a function of the depth $(z)$ in high resolution, ${ }^{39}$ from which one can estimate the nature of the film-substrate interface, the value of $d_{02}$ or $c$ (see Fig. 1), the total film thickness $(D)$ and the average density or porosity of the mesostructured films. $^{13}$

On the other hand, GISAXS measurements of the dried samples (after 30 days) were performed using a synchrotron source (a MiNaXS beam line, PETRA III) at an energy of $11.4 \mathrm{keV} .{ }^{40}$ The scattered beam was detected using a $2 \mathrm{D}$ detector (PILATUS $1 \mathrm{M}$, Dectris, having $981 \times 1043$ pixels and $172 \mu \mathrm{m}$ pixel size) by placing it about $171 \mathrm{~cm}$ away from the sample. For data collection, the incident angle $\alpha$ was kept at $0.25^{\circ}$, slightly greater than the critical angle $\alpha_{c}$ of the sample. The direct beam was stopped and the specular reflected beam was attenuated by two separate point-like beam stops to avoid

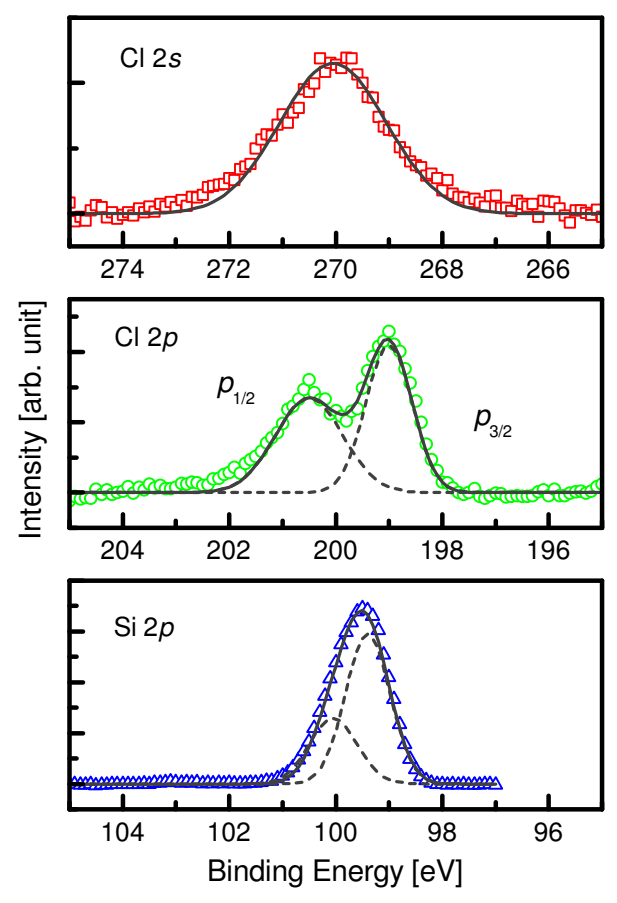

Fig. $2 \mathrm{XP}$ spectra of the $\mathrm{Cl} 2 s, \mathrm{Cl} 2 p$ and $\mathrm{Si} 2 p$ binding energy regions of the $\mathrm{Cl}-\mathrm{Si}(111)$ sample.

the saturation of the detector. The GISAXS data provide the value of $d_{02}$ and $d_{11}$ (see Fig. 1), from which one can extract the unit cell parameters $(b$ and $c)$ and the shape $\left(r_{l} / r_{s}\right)$ of the silica coated micelles. ${ }^{13}$

\section{Results and discussion}

\subsection{Chemical nature from XPS}

XPS scans for the Cl-Si(111) sample near $\mathrm{Si} 2 p, \mathrm{Cl} 2 p$ and $\mathrm{Cl} 2 s$ binding energies are shown in Fig. 2 . The peaks of the $\mathrm{Si}$ $2 p$ spectrum can be reproduced well by considering the $2 p_{3 / 2}$ and $2 p_{1 / 2}$ peaks, with a separation of $0.6 \mathrm{eV}$ and an area ratio of 2 , in agreement with the reported values. ${ }^{18}$ Similarly, the peaks of the $\mathrm{Cl} 2 p$ spectrum can be reproduced well, considering the two spin-orbit split peaks $\left(2 p_{3 / 2}\right.$ and $2 p_{1 / 2}$ with separation of about $1.5 \mathrm{eV})$. The presence of the $2 s, 2 p_{3 / 2}$ and $2 p_{1 / 2}$ peaks of $\mathrm{Cl}$ in the $\mathrm{Cl}-\mathrm{Si}(111)$ sample confirm the chlorination of the $\mathrm{Si}(111)$ surface. No significant peak for the silicon oxide layer is observed, indicating negligible oxidation of this surface. This $\mathrm{Cl}-\mathrm{Si}(111)$ substrate is henceforth referred to as the $\mathrm{Cl}-\mathrm{Si}$ sample.

\subsection{Hydrophilic/hydrophobic nature from CA}

The hydrophilic/hydrophobic nature of the differentlyterminated Si surfaces, in the macroscopic level, can be easily estimated from the CA measurements. It is necessary to men- 


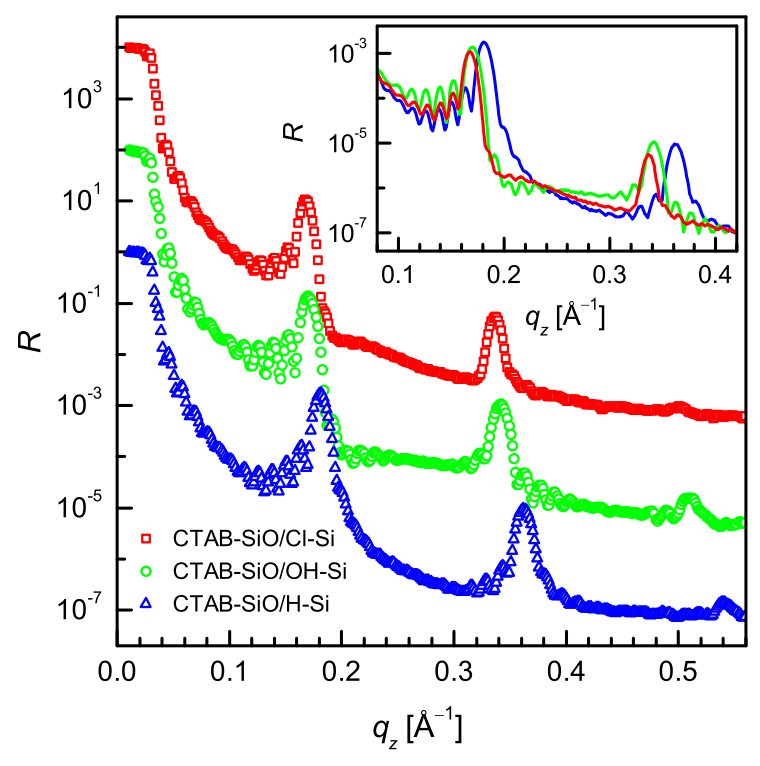

Fig. 3 XR data of CTAB-silica mesostructured thin films on differently-terminated Si substrates, measured initially (curves are shifted vertically for clarity). Inset: selected portion of the same data (without any shifting) to have better idea about the nature of variation, before and after the first Bragg peak, for the three samples.

tion that we have not measured the advancing and receding contact angles, which are more appropriate. What we measured is so-called as-placed CA, ${ }^{41}$ whose changes are qualitatively similar to those of the equilibrium CA. ${ }^{42}$ The CA value for the $\mathrm{Cl}-\mathrm{Si}$ surface is found to be about $27 \pm 5^{\circ}$, while those for the $\mathrm{OH}-\mathrm{Si}$ and $\mathrm{H}-\mathrm{Si}$ surfaces are found to be about $15 \pm 3^{\circ}$ and $64 \pm 4^{\circ}$, respectively. The footprint of water on the $\mathrm{Cl}-\mathrm{Si}$ surface is found to be about $4-5 \mathrm{~mm}$. The CA can provide the information about the adhesion, surface energy, or hydrophilicity/hyhrophobility (which is of our interest). ${ }^{24}$ The values of the $\mathrm{CA}$ for the $\mathrm{OH}-\mathrm{Si}$ and $\mathrm{H}-\mathrm{Si}$ surfaces correspond to the weakly hydrophilic and weakly hydrophobic nature of the surfaces, as expected. ${ }^{43}$ The value of $\mathrm{CA}$ for the $\mathrm{Cl}$-Si surface is very small compared to that of the H-Si surface, but large compared to that of the $\mathrm{OH}-\mathrm{Si}$ surface. This suggests that the $\mathrm{Cl}-\mathrm{Si}$ surface is weakly hydrophilic in nature.

\subsection{Hydrophilic/hydrophobic nature from structure}

\subsubsection{Interface and out-of-plane structures from $X R$}

The XR data of the CTAB-SiO/Cl-Si, CTAB-SiO/OH-Si and $\mathrm{CTAB}-\mathrm{SiO} / \mathrm{H}-\mathrm{Si}$ samples collected after preparation are shown in Fig. 3. Two strong and one very weak pseudo Bragg peaks are visible in all the curves, which correspond to the first, second and third orders of the (02) Bragg peak for the 2D-hexagonal mesostructure, suggesting the formation of well ordered 2D-hexagonal structure. The nature of the XR profile before and after the first Bragg peak for the CTAB-SiO/Cl-

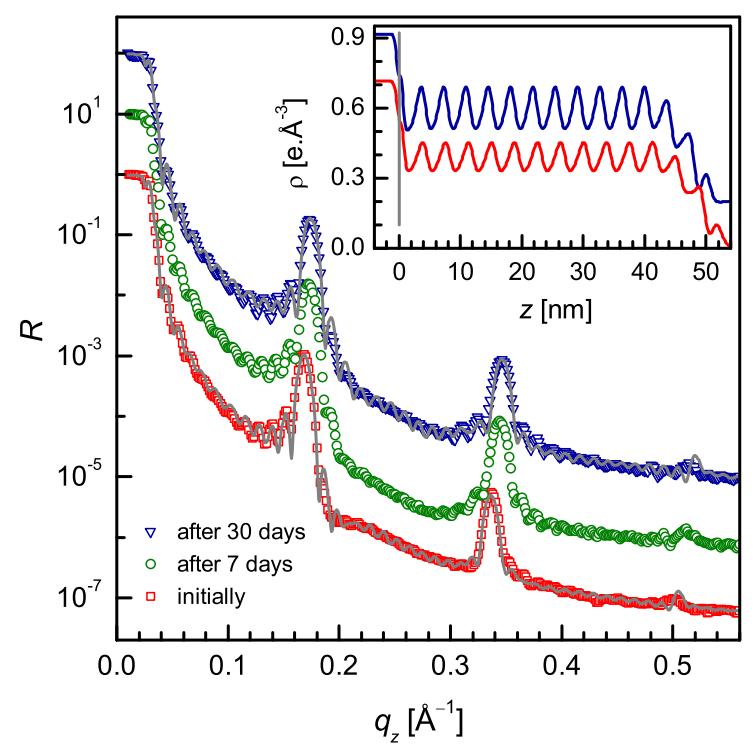

Fig. 4 XR data (different symbols) and analyzed curves (solid line) of the CTAB-silica mesostructured thin film on the Cl-Si substrate measured at different time intervals (curves are shifted vertically for clarity). Inset: corresponding EDPs measured initially (within a day) and finally (after 30 days).

Si sample is found to be intermediate to those of the CTAB$\mathrm{SiO} / \mathrm{OH}-\mathrm{Si}$ and CTAB-SiO/H-Si samples. This is clearly visible from the curves shown in the inset of Fig. 3. The peakheight of the first Bragg peak compared to the preceding dip is minimal for the CTAB-SiO/OH-Si sample and maximal for the CTAB-SiO/H-Si sample, while for the CTAB-SiO/Cl-Si sample it is in between. The fall after the first Bragg peak and before the second Bragg peak is sharp and then almost flat for the CTAB-SiO/OH-Si sample and very gradual for the CTAB$\mathrm{SiO} / \mathrm{H}-\mathrm{Si}$ sample, while for the CTAB-SiO/H-Si sample it is again in between, but more like the $\mathrm{CTAB}-\mathrm{SiO} / \mathrm{OH}-\mathrm{Si}$ sample. Kiessig fringes, which are the measure of the total film thickness, are very clearly observed for all the XR profiles before first Bragg peak. The values of $d$ (i.e. $d_{02}$ ) obtained from the (02) Bragg peak positions for the three films are tabulated in Table 1 with an accuracy better than $\pm 0.02 \mathrm{~nm}$. The value of $d$ for the film on $\mathrm{Cl}-\mathrm{Si}$ is large compared to that on $\mathrm{H}-\mathrm{Si}$ and even slightly larger than that on $\mathrm{OH}-\mathrm{Si}$.

$\mathrm{XR}$ data of the CTAB-SiO/Cl-Si sample collected in different time interval are shown in Fig. 4. There is no decrease in the Bragg peak intensity with time indicating no deterioration in the ordering of the film with drying. The Bragg peak positions however change with time but the change is mostly within 7 days. This is same for the change in the nature of the curve before and after the first Bragg peak. The change in the peak positions with time is associated with the decrease in the $d$ value with drying, while the change in the nature of the curve with time may be due to the change in the $d$ value and/or due to the change in the interface. To have a better idea about 
Table 1 Parameters such as the separation $(d)$ corresponding to the (02) Bragg peaks, the total film thickness $(D)$ and the number of repetitive layers $(N)$ for the three samples as obtained from the initially measured XR data.

\begin{tabular}{llll}
\hline Sample & $d(\mathrm{~nm})$ & $D(\mathrm{~nm})$ & $N$ \\
\hline CTAB-SiO/Cl-Si & 3.74 & 52.5 & 14 \\
CTAB-SiO/OH-Si & 3.70 & 48.4 & 13 \\
CTAB-SiO/H-Si & 3.47 & 54.0 & 15 \\
\hline
\end{tabular}

the structure of the film, especially about the film-substrate interface, the XR data have been analyzed using Parratt's formalism. ${ }^{44}$ For the analysis, a model oscillatory EDP, arising from periodic repetitions of two stacked layers is considered as before. ${ }^{13}$ One layer is made of cylindrical surfactant aggregates plus a silica wall and another layer is made of silica only, having different averaged electron density, thickness and roughness. In addition, special attention is paid to the initial layer through which the film is attached to the substrate, as it decides the nature of the substrate surface.

Based on this model, the different natures of the XR profiles (before and after the first Bragg peak) that we observed experimentally in Fig. 3 have been simulated (not shown here) considering only the different interfacial natures. Namely adsorption of the film onto the $\mathrm{H}-\mathrm{Si}$ substrate proceeds through hemicylindical micelles and that onto the $\mathrm{OH}-\mathrm{Si}$ substrate proceeds through the silica coated cylindrical micelles with the freshly prepared oxide surface, while that onto the Cl-Si substrate is directly through siica coated cylindical micelles. The best fit XR profiles for the CTAB-SiO/Cl-Si sample measured initially and finally are shown in Fig. 4 along with the corresponding EDPs in the inset. For the CTAB-SiO/Cl-Si sample measured initially, there is a dip after the substrate, which is gradual and wide. This is unlike the CTAB-SiO/OH-Si sample, where an intermediate plateau region corresponding to the silica layer is present, and also unlike the CTAB-SiO/H-Si sample, where the dip is quite sharp and narrow. The nature of the dip after the substrate in the CTAB-SiO/Cl-Si sample is more like that of the dip after the plateau region in the CTAB$\mathrm{SiO} / \mathrm{OH}-\mathrm{Si}$ sample. This suggests that the silica coated cylindrical micelles are directly adsorbed onto the $\mathrm{Cl}-\mathrm{Si}$ surface, which clearly indicates the hydrophilic nature of the Cl-Si surface. Correspondingly, the value of $d$ for the CTAB-SiO/Cl-Si sample is found to be similar to that for the CTAB-SiO/OH$\mathrm{Si}$ sample. With time the width and sharpness of the dip near the substrate for the CTAB-SiO/Cl-Si sample decreases and increases, respectively, which is related to the change in the shape of the micelles from nearly circular to more elliptical, mainly due to the drying of the film that is strongly attached to the substrate.

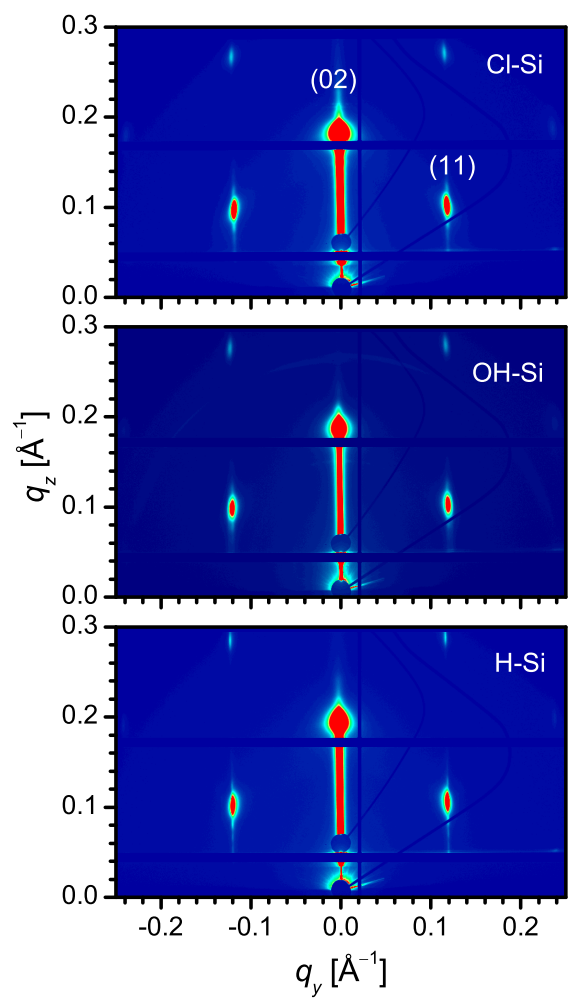

Fig. 5 GISAXS patterns of the CTAB-silica dried films on differently-terminated Si substrates, showing (11) and (02) Bragg spots of the compressed 2D-hexagonal structure.

\subsubsection{In-plane and out-of-plane structures from GISAXS}

The XR results provide information on the out-of-plane separation between the micelles in the films and the attachments of the films to the substrates. Such information allows us to predict possible structures of the films but to ascertain the prediction, structural information along both (out-of-plane and in-plane) directions, which can be obtained from GISAXS results, is very important. GISAXS patterns of the dried CTABsilica mesostructured films on differently-terminated Si substrates are shown in Fig 5. (02) and (11) Bragg spots, signatures of a compressed 2D-hexagonal structure, are evident in all the patterns. In-plane $(b)$ and out-of-plane $\left(\mathrm{c}_{f}\right)$ structural

Table 2 In-plane (b) and out-of-plane (c) unit cell parameters of the compressed 2D-hexagonal structure, the ratio of the semi-major and semi-minor axis $\left(r_{l} / r_{s}\right)$ corresponding to the equivalent ellipse for the different films on differently-terminated Si substrates. The subscripts $\mathrm{i}$ and $\mathrm{f}$ represent parameters corresponding to the initial and final time of measurements, respectively.

\begin{tabular}{llllll}
\hline Sample & $b(\mathrm{~nm})$ & $c_{\mathrm{i}}(\mathrm{nm})$ & $c_{\mathrm{f}}(\mathrm{nm})$ & $\left(r_{l} / r_{s}\right)_{\mathrm{i}}$ & $\left(r_{l} / r_{s}\right)$ \\
\hline fTAB-SiO/Cl-Si & 5.27 & 7.48 & 7.28 & 1.22 & 1.25 \\
CTAB-SiO/OH-Si & 5.25 & 7.38 & 7.24 & 1.23 & 1.26 \\
CTAB-SiO/H-Si & 5.26 & 6.94 & 6.92 & 1.31 & 1.32 \\
\hline
\end{tabular}


parameters for all the dried films, extracted from GISAXS spots wuth an accuracy better than $\pm 0.02 \mathrm{~nm}$, are listed in Table 2. The value of $c_{i}(=2 d)$ for all three films obtained from the initial XR measurements (Table 1) are also listed in Table 2. The distance between the (11) planes for the films on all the substrates is the same. This means that although the initial attachment of the films and the out-of plane distance of the micelles are different on differently-terminated substrates, the in-plane distance between the micelles is the same. The ratios of the semi-major and semi-minor axis $\left(r_{l} / r_{s}\right)$ which is estimated from $\sqrt{3} /(c / b)$ are listed in Table 2. The value of $r_{l} / r_{s}$ for the film on $\mathrm{H}-\mathrm{Si}$ is more compared to that on $\mathrm{OH}-\mathrm{Si}$, as reported earlier. ${ }^{13}$ The value of $r_{l} / r_{s}$ for the dried film on the $\mathrm{Cl}-\mathrm{Si}$ is similar to that for the film on the $\mathrm{OH}-\mathrm{Si}$, while the value for the as prepared film on $\mathrm{Cl}-\mathrm{Si}$ is even smaller compared to that for the film on $\mathrm{OH}-\mathrm{Si}$. This indicates that the micelles are most elliptical on the H-Si substrate and least elliptical (i.e. more circular) on the Cl-Si substrate during the initial stages of the film formation.

\subsection{Hydrophilic-like nature and possible origin}

An intermediate wetting behavior of the Cl-Si surface compared to the $\mathrm{OH}-\mathrm{Si}$ and $\mathrm{H}-\mathrm{Si}$ surfaces was observed from the CA measurements, which was more similar to that of the OH$\mathrm{Si}$ surface, i.e. the $\mathrm{Cl}-\mathrm{Si}$ surface is hydrophilic-like in nature from a macroscopic point of view. The same intermediate hydrophilic-like nature of the Cl-Si surface is also obtained from the XR data analysis (i.e. from the information of the film-substrate interface). It can be noted that the adsorption of the film with the substrate takes place on the molecular level and the formation of a particular interface depends on the nature of the substrate surface on the molecular or microscopic level. The information on the shape $\left(r_{l} / r_{s}\right)$ of the micelles inside the films in the initial stages, which is induced by the substrate surface nature, however, suggests that the Cl-Si surface is hydrophilic, similar to that of the $\mathrm{OH}-\mathrm{Si}$ surface or even stronger. So from both the macroscopic and microscopic point of view, as shown schematically in Fig. 6, the Cl-Si surface is weakly hydrophilic or hydrophilic-like in nature.

In the wet chemical process, the $\mathrm{H}$ atoms of the $\mathrm{H}-\mathrm{Si}$ surface are exchanged with $\mathrm{Cl}$ atoms. The relatively strong electronegativity of $\mathrm{Cl}$ atom, listed in Table 3, helps to replace the $\mathrm{H}$ atom, leaving the surface $\mathrm{Cl}$-terminated. On the other hand, large atomic size of $\mathrm{Cl}$ prevents such replacement due to steric-hindrance, especially for the $\mathrm{Si}(001)$ surface (where two $\mathrm{H}$-saturated dangling bonds corresponding to a single $\mathrm{Si}$ atom need to replace) and also for the rough surface (where the access to H-saturated dangling bonds near the dip is restricted). A high atomic roughness of the $\mathrm{H}-\mathrm{Si}(100)$ surface compared to the $\mathrm{H}-\mathrm{Si}(111)$ surface is usually observed. ${ }^{17}$ Thus, both steric-hindrance and high roughness make the H-Si(100) surface relatively difficult to terminate with $\mathrm{Cl}$ compared to the $\mathrm{H}-\mathrm{Si}(111)$ surface. This is also consistence with the surface

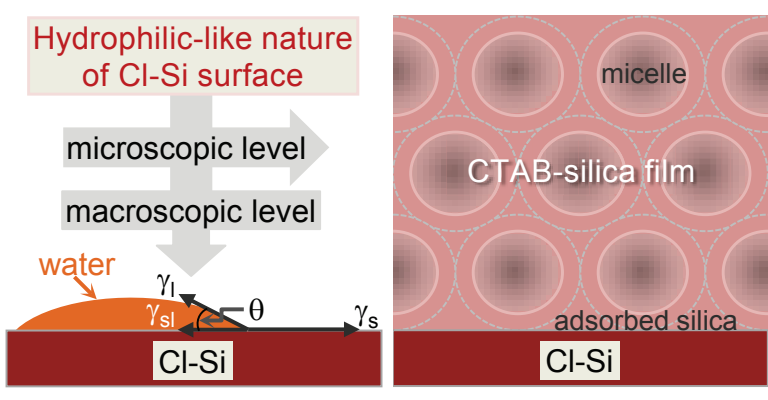

Fig. 6 Schematic of the wetting with water (on a mm length scale) and the structure of the CTAB-silica film (on a nm length scale) on the hydrophilic-like Cl-Si surface as predicted from the CA, XR and GISAXS measurements. The surface free energy of solid $\left(\gamma_{\mathrm{s}}\right)$, liquid $\left(\gamma_{1}\right)$ and interfacial energy $\left(\gamma_{\mathrm{sl}}\right)$ dictates the $\mathrm{CA}(\theta)$ value and the wetting. The adsorbed silica dictates the shape of micelles and the structure of the film.

roughness value of the $\mathrm{Cl}-\mathrm{Si}(111)$ and $\mathrm{Cl}-\mathrm{Si}(100)$ samples (about 0.54 and $0.96 \mathrm{~nm}$, respectively) as obtained from the analysis of the XR data (not shown here). Even the Si(111) surface is not completely terminated with $\mathrm{Cl}$ under most experimental conditions. A few $\mathrm{H}$ atoms are never replaced with $\mathrm{Cl}$ atoms and a few $\mathrm{Cl}$ atoms are further replaced with $\mathrm{O}$ atoms. In our case, the presence of the $\mathrm{Cl}\left(2 s, 2 p_{1 / 2}\right.$ and $\left.2 p_{3 / 2}\right)$ and $\mathrm{Si}\left(2 p_{1 / 2}\right.$ and $\left.2 p_{3 / 2}\right)$ peaks and the absence of any significant silicon oxide peak in the XPS scans (Fig. 2) clearly suggests that the $\mathrm{Cl}-\mathrm{Si}(111)$ surface is mostly Cl-terminated.

The hydrophilic nature of the Cl-Si surface compared to the H-Si surface can be well realized considering the polarnonpolar nature. The electronegativity difference between $\mathrm{Cl}$ and Si (1.26) is greater than that between $\mathrm{H}$ and Si (0.30), which makes the former polar and hydrophilic, while the latter is nonpolar and hydrophobic. The electronegativity difference between $\mathrm{O}$ and $\mathrm{Si}$ (1.54) is quite high, but still the $\mathrm{O}-\mathrm{Si}$ surface is not quite hydrophilic; this is because each $\mathrm{O}$ atom is connected to two $\mathrm{Si}$ atoms resulting in a decrease in the value of the effective electronegativity difference. The very strong electronegativity of the $\mathrm{OH}$ group makes the $\mathrm{OH}-\mathrm{Si}$ surface highly polar and hydrophilic. The high hydrophilicity of the $\mathrm{OH}-\mathrm{Si}$ surface is also related to the hydrogen bonding ability of this surface with the water molecules, which is not possi-

Table 3 The covalent atomic radii $\left(R_{a}\right)$, the electronegativity $(\delta)$ on Pauling scale, the bond-energy $\left(D_{B}\right)$ with $\mathrm{Si}$ and the bond-length $\left(L_{B}\right)$ with $\mathrm{Si}$ for different elements, as obtained from online sites. $^{45,46}$

\begin{tabular}{lllll}
\hline Element & $R_{a}(\AA)$ & $\delta$ & $D_{B}(\mathrm{~kJ} / \mathrm{mole})$ & $L_{B}(\AA)$ \\
\hline $\mathrm{Si}$ & 1.11 & 1.90 & 222 & 2.33 \\
$\mathrm{Cl}$ & 0.99 & 3.16 & 381 & 2.02 \\
$\mathrm{O}$ & 0.73 & 3.44 & 452 & 1.63 \\
$\mathrm{H}$ & 0.37 & 2.20 & 318 & 1.48 \\
\hline
\end{tabular}


ble for the $\mathrm{Cl}$-Si surface. Due to the strong electronegativity of $\mathrm{Cl}$, however, it is able to attract water molecules and other hydrophilic particles even better, as observed for the CTABsilica film. The lower wettability of the completely converted $\mathrm{Cl}-\mathrm{Si}$ surface compared to the $\mathrm{OH}-\mathrm{Si}$ surface is likely to be due to the absence of hydrogen bonding in the former. In reality, the Cl-Si surface is not completely (though mostly) terminated with $\mathrm{Cl}$. A few different atoms that may present are distributed uniformly, making the Cl-Si surface homogeneous and hydrophilic-like but comparatively less hydrophilic as observed from the CA measurement. Silica coated cylindrical micelles are adsorbed through their silica parts onto this homogeneous and hydrophilic-like Cl-Si surface. However, the presence of different atoms probably restricts the length of the cylinder, which helps the micelles above to relax and to form slightly more circular cylinders compared to those on the $\mathrm{OH}-\mathrm{Si}$ surface as observed from the $r_{l} / r_{s}$ values at the initial stages. The attachment of the silica layers with the $\mathrm{Cl}-\mathrm{Si}$ surface is, however, quite strong, which creates strong asymmetric stress on the micelles while drying, especially near the substrate.

\section{Conclusions}

The wettability or hydrophilicity of a Cl-terminated Si surface prepared by a wet chemical process was examined. An XPS study confirms the chlorination of the $\mathrm{Si}(111)$ surface. The $\mathrm{CA}$ of the $\mathrm{Cl}-\mathrm{Si}(111)$ surface was found to be intermediate between $\mathrm{OH}-\mathrm{Si}$ and $\mathrm{H}-\mathrm{Si}$, but closer to that of the $\mathrm{OH}-\mathrm{Si}$ surface, indicating the hydrophilic-like nature of the $\mathrm{Cl}$-Si surface on the macroscopic level. A structural study of the CTABsilica film on the $\mathrm{Cl}-\mathrm{Si}$ substrate suggests the formation of a well-ordered, compressed 2D-hexagonal structure, similar to those observed for the films on the OH-Si and H-Si substrates. Their structural stability suggests quite a strong interaction between the film and the substrate and not much change in the substrate surface nature with time. Silica molecules adsorbed onto the Cl-Si surface to form a silica layer, similar to that observed for the hydrophilic $\mathrm{OH}-\mathrm{Si}$ surface and unlike that for the hydrophobic H-Si surface, where the hydrophobic parts of the CTAB molecules adsorbed to form hemicircular micelles. Also, the shape of the micelles (which is induced through initial attachment) on the Cl-Si surface is nearly circular, similar to that observed on the OH-Si surface (less elliptical) and unlike that observed on the $\mathrm{H}-\mathrm{Si}$ surface (more elliptical). Both of these results suggest a hydrophilic-like nature of the $\mathrm{Cl}-\mathrm{Si}$ surface even on the molecular or microscopic level, which can be explained by considering the strong electronegativity of $\mathrm{Cl}$, with some counter-balance from the lack of hydrogen bonding and/or the lack of complete $\mathrm{Cl}$ termination.

\section{Acknowledgements}

The authors thank Dr. S. Roth, Prof. N. Roy and Prof.
M. Mukherjee for their support in GISAXS, contact angle and XPS measurements, respectively. The financial support received from Saha Institute of Nuclear Physics under DSTDESY project to carry out GISAXS experiments at PETRA III is thankfully acknowledged.

\section{References}

1 J. Zasadzinski, R. Viswanathan, L. Madsen, J. Garnaes and D. Schwartz, Science, 2004, 263, 1726.

2 M. J. Rosen, Surfactants and Interfacial Phenomena, John Wiley, NJ, 2004.

3 M. M. Stevens and J. H. George, Science, 2005, 310, 1135.

4 S. Koynov, M. S. Brandt and M. Stutzmann, Appl. Phys. Lett., 2006, 88, 203107.

5 J. K. Bal and S. Hazra, Phys. Rev. B, 2007, 75, 205411.

6 J. K. Bal and S. Hazra, Phys. Rev. B, 2009, 79, 155412.

7 J. K. Bal, S. Kundu and S. Hazra, Phys. Rev. B, 2010, 81, 045404.

8 J. K. Bal, S. Kundu and S. Hazra, Chem. Phys. Lett., 2010, 500, 90.

9 J. K. Bal, S. Kundu and S. Hazra, Eur. Phys. J. E, 2012, 35, 79.

10 H. F. Okorn-Schmidt, IBM J. Res. Dev., 1999, 43, 351.

11 X. G. Zhang, Electrochemistry of Silicon and its Oxide, Kluwer Academic, New York, 2004.

12 Y. Dan, K. Seo, K. Takei, J. H. Meza, A. Javey and K. B. Crozier, Nano Lett., 2011, 11, 2527.

13 P. Chatterjee, S. Hazra and H. Amenitsch, Soft Matter, 2012, 8, 2956.

14 K. A. Perrine and A. V. Teplyakov, Chem. Soc. Rev., 2010, 39, 3256.

15 F. A. Soria, E. M. Patrito and P. Paredes-Oliverai, Langmuir, 2011, 27, 2613.

16 S. Rivillon, Y. J. Chabal, L. J. Webb, D. J. Michalak, N. S. Lewis, M. D. Halls and K. Raghavachari, J. Vac. Sci. Technol. A, 2005, 23, 1100.

17 S. Rivillon, F. Amy, Y. J. Chabal and M. M. Frank, Appl. Phys. Lett., 2004, 85, 2583.

18 A. Bansal, X. Li, S. I. Yi, W. H. Weinberg and N. S. Lewis, J. Phys. Chem. $B, 2001, \mathbf{1 0 5}, 10266$

19 S. Rivillon, R. T. Brewer and Y. J. Chabal, Appl. Phys. Lett., 2005, 87, 173118.

20 L. J. Webb, D. J. Michalak, J. S. Biteen, B. S. Brunschwig, A. S. Y. Chan, D. W. Knapp, H. M. Meyer, E. J. Nemanick, M. C. Traub and N. S. Lewis, J. Phys. Chem. B, 2006, 110, 23450.

21 C. Y. Ruan, V. A. Lobastov, F. Vigliotti, S. Chen and A. H. Zewail, Science, 2004, 304, 80.

22 P. L. Silvestrelli, F. Toigo and F. Ancilotto, J. Phys. Chem. B, 2006, 110, 12022.

23 P. L. Silvestrelli, F. Toigo and F. Ancilotto, J. Phys. Chem. C, 2009, 113, 17124.

24 J. Drelich, E. Chibowski, D. D. Meng and K. Terpilowski, Soft Matter, 2011, 7, 9804.

25 P. G. de Gennes, Rev. Mod. Phys., 1985, 57, 827.

26 R. Tadmor, P. Bahadur, A. Leh, H. E. N'guessan, R. Jaini and L. Dangi, Phys. Rev. Lett., 2009, 103, 266101.

27 R. Tadmor, Soft Matter, 2011, 7, 1577.

28 D. A. Doshi, A. Gibaud, V. Goletto, M. Lu, H. Gerung, B. Ocko, S. M. Han and C. J. Brinker, J. Am. Chem. Soc., 2003, 125, 11646.

29 L. Nicole, C. Boissiere, D. Grosso, A. Quach and C. Sanchez, J. Mater. Chem., 2005, 15, 3598.

30 M. Matheron, T. Gacoin and J.-P. Boilot, Soft Matter, 2007, 3, 223.

31 I. K. Robinson and D. J. Tweet, Rep. Prog. Phys., 1992, 55, 599.

$32 X$-Ray and Neutron Reflectivity: Principles and Applications, ed. J. Daillant and A. Gibaud, Springer, Paris, 1999.

33 A. Naudon and D. Babonneau, Z. Metallkd., 1997, 88, 596.

34 S. Hazra, A. Gibaud, A. Desert, C. Sella and A. Naudon, Physica B, 2000, 283, 97. 
35 A. Gibaud, D. Grosso, B. Smarsly, A. Baptiste, J. F. Bardeau, F. Babonneau, D. A. Doshi, Z. Chen, C. J. Brinker and C. Sanchez, J. Phys. Chem. $B, 2003,107,6114$.

36 A. J. Allen, J. Am. Ceram. Soc., 2005, 88, 1367.

37 S. Hazra, A. Gibaud and C. Sella, J. Appl. Phys., 2007, 101, 113532.

38 S. Hazra, Appl. Surf. Sci., 2006, 253, 2154.

39 J. K. Bal and S. Hazra, Phys. Rev. B, 2009, 79, 155405.

40 S. V. Roth, G. Herzog, V. Körstgens, A. Buffet, M. Schwartzkopf, J. Perlich, M. M. A. Kashem, R. Döhrmann, R. Gehrke, A. Rothkirch, K. Stas- sig, W. Wurth, G. Benecke, C. Li, P. Fratzl, M. Rawolle and P. MüllerBuschbaum, J. Phys.: Condens. Matter, 2011, 23, 254208.

41 R. Tadmor and P. S. Yadav, J. Colloid Interf. Sci., 2008, 317, 241.

42 R. Tadmor, Langmuir, 2004, 20, 7659.

43 J. K. Bal, S. Kundu and S. Hazra, Chem. Phys., 2012, 406, 72.

44 L. G. Parratt, Phys. Rev., 1954, 95, 359.

45 Periodic Table of Elements, http://www.ptable.com.

46 Wired Chemist, http://www.wiredchemist.com. 